\title{
Shikonin potentiates paclitaxel antitumor efficacy in esophageal cancer cells via the apoptotic pathway
}

\author{
WENZHEN DU ${ }^{1 *}$, XIAOHONG HAO ${ }^{2 *}$, ZHILI YUAN $^{1}$, YING WANG $^{3}$, XUEGUANG ZHANG ${ }^{4}$ and JIE LIU $^{1}$ \\ Departments of ${ }^{1}$ Gastroenterology, and ${ }^{2}$ Hematology and Oncology, Yantai Yeda Hospital, Yantai, Shandong 264000; \\ Departments of ${ }^{3}$ Otolaryngology and ${ }^{4}$ Neurosurgery, Liaocheng People's Hospital, Liaocheng, Shandong 252000, P.R. China
}

Received October 2, 2018; Accepted June 7, 2019

DOI: $10.3892 / \mathrm{ol} .2019 .10662$

\begin{abstract}
Shikonin is a natural naphthoquinone pigment that can suppress the growth of a number of cancer cell types. Paclitaxel is an antineoplastic chemotherapy drug, which is used for the treatment of various types of solid tumor cancer. However, acquired paclitaxel resistance results in the failure of therapy, and consequent metastasis and relapse. The aim of the present study was to investigate whether shikonin can sensitize esophageal cancer cells to paclitaxel-treatment and to elucidate the underlying mechanisms. The biological effects of these two agents on esophageal cancer cell lines KYSE270 and KYSE150 were investigated by MTT assay, cell cycle analysis, Annexin-V apoptosis assay, western blotting and reverse transcription-quantitative polymerase chain reaction. The results demonstrated that shikonin could significantly increase the cell growth inhibition effect induced by paclitaxel in the examined cell lines $(\mathrm{P}<0.001)$. The addition of shikonin to paclitaxel promoted cancer cell mitotic arrest and induced significantly higher levels of cell apoptosis. Notably, the mRNA and protein levels of Bcl-2 were downregulated, while p53 was upregulated in KYSE270 and KYSE150 cells following combined treatment. In summary, shikonin can sensitize esophageal cancer cells to paclitaxel-treatment by promoting cell mitotic arrest and reinforcing the susceptibility of esophageal cancer cells to apoptosis induced by paclitaxel, which is potentially associated with altered levels of Bcl-2 and p53.
\end{abstract}

\section{Introduction}

Esophageal cancer (EC) is the eigth most common malignancy and the seventh leading cause of cancer-associated

Correspondence to: Dr Jie Liu, Department of Gastroenterology, Yantai Yeda Hospital, 11 Taishan Road, Yantai, Shandong 264000, P.R. China

E-mail: liujie838514@163.com

*Contributed equally

Key words: esophageal cancer, shikonin, paclitaxel, KYSE270 cells, mitotic arrest, apoptosis, Bcl-2, p53 mortality worldwide (1). There are two main histological types of EC, including esophageal squamous cell carcinoma (ESCC) and esophageal adenocarcinoma (2). Nearly $50 \%$ of EC cases worldwide occur in China, and EC had the third highest incidence rate and fourth higher mortality rate in China according to the Cancer Statistics in China for 2015 (3). Additionally, the majority of EC cases in China are ESCC, which accounts for $>90 \%$ of new EC cases (4). Despite advancements in diagnostic and therapeutic techniques over the past three decades, the prognosis of ESCC remains poor, with a 5-year survival rate of $<10 \%$ (5). Thus, the search for an effective therapy for controlling this devastating disease is ongoing. Since ESCC cells avoid apoptosis, novel therapeutic strategies should be investigated for the induction of apoptosis in ESCC cells.

The antineoplastic drug paclitaxel is a microtubule-targeting agent widely utilized in the clinic for the treatment of patients with ESCC and several other types of solid tumor (6). Paclitaxel blocks cells in the G2/M phase of the cell cycle by stabilizing microtubules (7). As a result, chromosomes fail to achieve the metaphase spindle configuration, which further interferes with chromosome separation, leading to apoptosis (8-10). Paclitaxel has offered substantial improvements in patient survival; however, numerous patients acquire resistance to paclitaxel, which reduces the anti-cancer effect of paclitaxel (11). Therefore, it is important to identify non-cytotoxic drugs to increase paclitaxel sensitivity.

Shikonin, a natural product isolated from the plant Lithospermum erythrohizon, which has long been used in Traditional Chinese Medicine, is understood to act on a variety of molecular targets associated with carcinogenesis, including pyruvate kinase M2 (12). Previous studies have demonstrated that shikonin exhibits significant antitumor potential by inducing apoptosis and necroptosis in cancer cell lines of various types, including breast cancer, hepatocellular carcinoma, leukemia, glioma and osteosarcoma (13-17). These findings indicate that shikonin exhibits therapeutic promise for the treatment of cancer. In addition, shikonin can enhance the sensitivity of cells to the anticancer agents in various types of cancer. For instance, shikonin has been identified to enhance adriamycin antitumor effects in lung cancer (18). Additionally, there is a potential for combined synergistic effects of shikonin and arsenic trioxide against human hepatocellular carcinoma (19). 
The present study identified that the combination of paclitaxel with shikonin has significantly improved effects on esophageal cancer in vitro. The present study provides molecular insights into the apoptosis process involved in combined paclitaxel and shikonin treatment by detecting alterations of p53 and Bcl-2, and the data indicates that the combination of paclitaxel and shikonin may be a promising alternative chemotherapeutic strategy for esophageal cancer.

\section{Materials and methods}

Cell culture and chemicals. The ESCC cell lines KYSE270 and KYSE150, kindly provided by Dr Hui Zhang (Sun Yat-Sen University Cancer Center, Guangzhou, China), were cultured in RPMI-1640 medium (Thermo fisher Scientific, Inc., Waltham, MA, USA), supplemented with $10 \%(\mathrm{v} / \mathrm{v})$ fetal bovine serum (Thermo fisher Scientific, Inc.) and $1 \%$ penicillin/streptomycin (Thermo Fisher Scientific Inc.). Cells were incubated at $37^{\circ} \mathrm{C}$ in a $5 \% \mathrm{CO}_{2}$ humidified atmosphere. Paclitaxel, purchased from the National Institute for the Control of Pharmaceutical and Biological Products (Beijing, China), and shikonin, purchased from Sigma-Aldrich; Merck KGaA (Darmstadt, Germany), were dissolved in DMSO.

MTT assay. MTT cytotoxicity assay was performed according to manufacturer's protocol (Sigma-Aldrich; Merck KGaA). Briefly, KYSE270 and KYSE150 cells were plated in 96-well plates, at a density of 2,000 cells per well in $100 \mu \mathrm{l}$ medium. Following overnight culture, cells were treated with different treatments for four days, including DMSO, paclitaxel (10, 100 and $200 \mathrm{nM})$, shikonin $(1 \mu \mathrm{M})$ and paclitaxel $(100 \mathrm{nM})$ combined with shikonin $(1 \mu \mathrm{M})$. On days $1,2,3$ and 4, $10 \mu \mathrm{l}$ MTT solution was added to each well and incubated at room temperature for $3 \mathrm{~h}$. Subsequently, $100 \mu \mathrm{l}$ DMSO was added to each well and the plates were incubated overnight. The absorbance was then measured using SpectraMax 190 (Molecular Devices, LLC, Sunnyvale, CA, USA) at a wavelength of $490 \mathrm{~nm}$. The experiment was performed three times.

Cell cycle analysis and Annexin-V apoptosis assay by flow cytometry. KYSE 270 and KYSE150 cells were plated into 12 -well plates at $1 \times 10^{5} /$ well, and treated with DMSO, shikonin $(1 \mu \mathrm{M})$, paclitaxel $(100 \mathrm{nM})$ or a combination of shikonin $(1 \mu \mathrm{M})$ and paclitaxel $(100 \mathrm{nM})$ at $37^{\circ} \mathrm{C}$ in a $5 \%$ $\mathrm{CO}_{2}$ humidified atmosphere. Following $24 \mathrm{~h}$, the cells were collected and fixed in $70 \%$ ethanol at $-20^{\circ} \mathrm{C}$ overnight. Subsequently, the cells were washed twice with ice-cold PBS. For cell cycle analysis, the cells were incubated with RNase A $(100 \mu \mathrm{g} / \mathrm{ml}$; Sigma-Aldrich; Merck KGaA) and propidium iodide (PI; $50 \mu \mathrm{g} / \mathrm{ml}$; Sigma-Aldrich; Merck KGaA) at room temperature for $30 \mathrm{~min}$. The cells were then analyzed by flow cytometry with a BD FACSCalibur (BD Biosciences, San Jose, CA, USA). For the Annexin-V apoptosis assay, the cells were double stained with PI and Annexin-V-FITC according to the manufacturer's protocol (Nanjing KeyGen Biotech, Co., Ltd., Nanjing, China). The cells were then immediately analyzed on BD FACSCalibur flow cytometer. All FACS data were analyzed with FlowJo v7.6 software (FlowJo, LLC, Ashland, OR, USA). All experiments were repeated three times.
Reverse transcription-quantitative PCR analysis. KYSE270 cells were collected for RNA isolation at $48 \mathrm{~h}$ following treatment with DMSO, shikonin $(1 \mu \mathrm{M})$, paclitaxel $(100 \mathrm{nM})$ or a combination of shikonin $(1 \mu \mathrm{M})$ and paclitaxel $(100 \mathrm{nM})$ at $37^{\circ} \mathrm{C}$ in a $5 \% \mathrm{CO}_{2}$ humidified atmosphere. Total RNA was extracted from KYSE270 cells using TRIzol ${ }^{\circledR}$ (Invitrogen; Thermo Fisher Scientific, Inc., Waltham, MA, USA), according to the manufacturer's protocol. Total RNA $(1 \mu \mathrm{g})$ was reverse transcribed into cDNA using MMLV reverse transcriptase according to the manufacturer's protocol. (Invitrogen; Thermo Fisher Scientific, Inc.). Bcl-2 and p53 gene expression was analyzed by quantitative PCR in the presence of SYBR Green Master mix (Bio-Rad Laboratories, Inc., Hercules, CA, USA), with the following primers: Bcl-2 forward, 5'-CGACGACTTCTCCCGCCGCTACCGC-3' and reverse, 5'-CCGCATGCTGGGGCCGTACAGTTCC-3'; p53 forward, 5'-TGTGGGATGGGGTGAGATTTC-3' and reverse, 5'-CTGTTGGTCGGTGGGTTG-3'. GAPDH was amplified as an endogenous control using the following primers: forward, 5'-ACGGATTTGGTCGTATTGGG-3' and reverse, 5'-TGATTTTGGAGGGATGTCGC-3'. The relative expression levels of Bcl-2 and p53 compared were GAPDH in each sample were calculated according to $2^{-\Delta \Delta \mathrm{Cq}}$ method (20). The thermocycling conditions were: i) An initial $95^{\circ} \mathrm{C}$ for $10 \mathrm{~min}$; ii) 40 cycles of denaturation at $95^{\circ} \mathrm{C}$ for $30 \mathrm{sec}$, annealing with primer at $57^{\circ} \mathrm{C}$ for $30 \mathrm{sec}$ and extension at $72^{\circ} \mathrm{C}$ for $30 \mathrm{sec}$; and ii) final extension at $72^{\circ} \mathrm{C}$ for $10 \mathrm{~min}$. The experiments were performed three times.

Western blot analysis. KYSE270 and KYSE150 cells were collected for protein extraction at $48 \mathrm{~h}$ following the indicated treatments. The cell lysates were prepared in RIPA buffer (Thermo Fisher Scientific, Inc.). Following protein quantification with a BCA kit (Bio-Rad Laboratories, Inc.), $20 \mu \mathrm{g}$ protein was loaded per lane, separated by $8 \%$ SDS-PAGE, and transferred to a PVDF membrane. Following blocking with $5 \%$ non-fat milk for $1 \mathrm{~h}$ at room temperature, the membranes were incubated with anti-Bcl-2 (cat. no. 4223T; 1:1,000), anti-cleaved caspase 3 (cat. no. 9664T; 1:1,000), anti-cleaved poly (ADP-ribose) polymerase (PARP; cat. no. 5625T; 1:1,000), anti-p53 (cat. no. 2527T; 1:1,000) and anti-GAPDH (cat. no. 2118S; 1:1,000) antibodies overnight at $4^{\circ} \mathrm{C}$. The membranes were then washed with TBS-T (TBS with $0.05 \%$ Tween-20) three times for $5 \mathrm{~min}$ each time. Subsequently, the membranes were incubated with a horseradish peroxidase-conjugated anti-rabbit secondary antibody (cat. no. 7074S; 1:2,000) at room temperature for $1 \mathrm{~h}$. All antibodies were purchased from Cell signaling Technology, Inc., (Danvers, MA, USA). After washing three times, the signals were detected using an ECL kit (Bio-Rad Laboratories, Inc., Hercules, CA, USA).

Statistical analysis. All data are presented as the mean \pm standard error of the mean. The data from two groups or multiple groups were compared using a Student's t-test or one-way ANOVA with Dunnett's multiple comparisons test. All Statistical analysis was implemented using SPSS 13.0 software (SPSS, Inc., Chicago, IL, USA). All experiments were repeated at least three times. $\mathrm{P}<0.05$ was considered to indicate a statistically significant difference. 

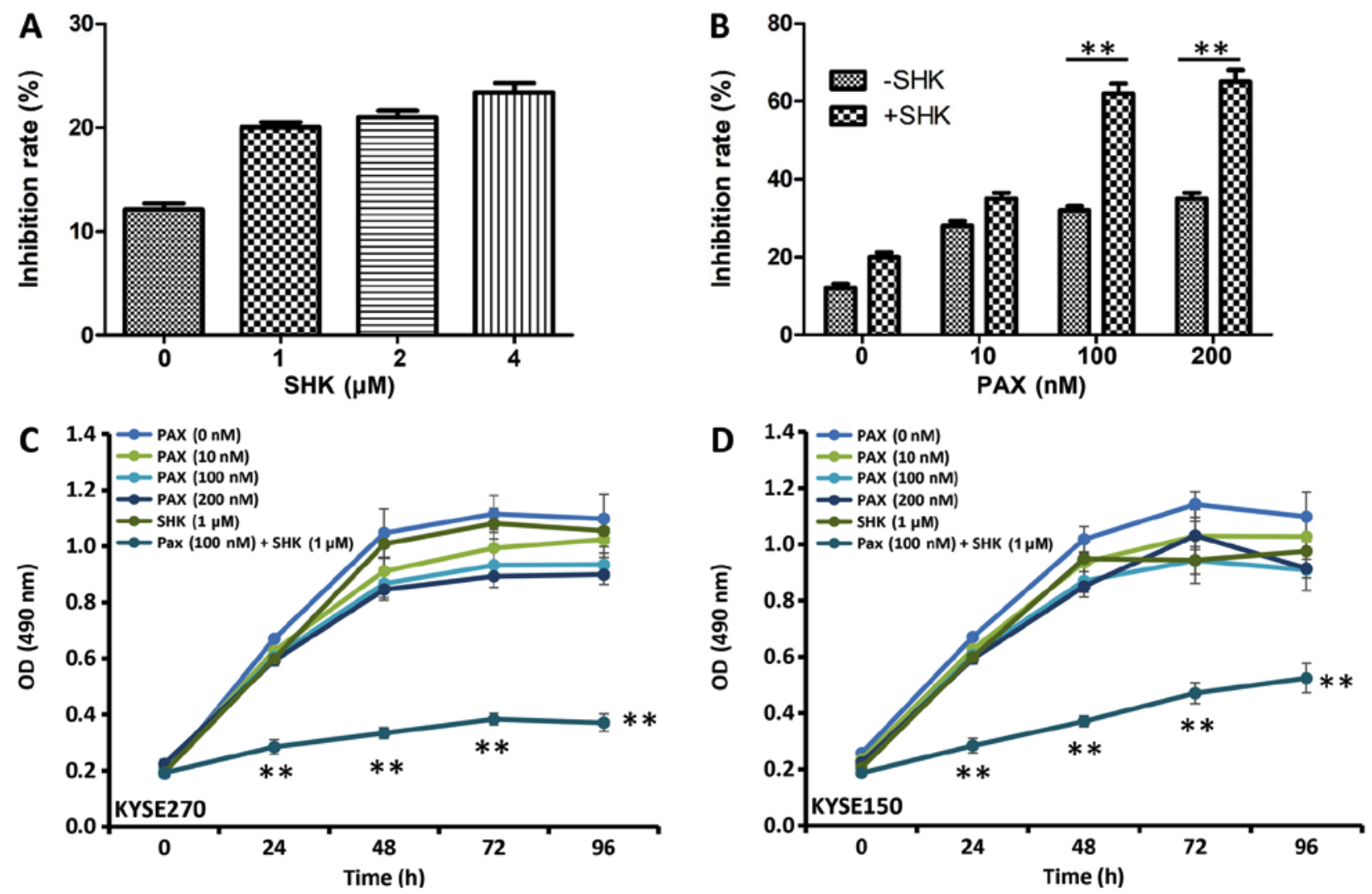

Figure 1. Effects of shikonin and paclitaxel on esophageal squamous cell carcinoma cell growth. KYSE270 cell growth inhibition was determined $48 \mathrm{~h}$ following treatment with increasing doses of (A) shikonin, (B) paclitaxel or a combination of shikonin $(1 \mu \mathrm{M})$ and paclitaxel by MTT assay. Data were analyzed by Student's t-test. ${ }^{* *} \mathrm{P}<0.001$. (C) KYSE270 and (D) KYSE150 cell growth at different time points following indicated treatment as measured by the MTT assay. Data were analyzed by one-way ANOVA with Bonferroni's correction, and presented as the mean \pm standard error or the mean from three independent experiments. ${ }^{* *} \mathrm{P}<0.001$ vs. PAX $(100 \mu \mathrm{M})+$ SHK $(1 \mathrm{mM})$. PAX, paclitaxel; SHK, shikonin; OD, optical density.

\section{Results}

Shikonin significantly enhances the cytotoxic effects of paclitaxel in KYSE270 cells. To determine the cytotoxic effect of shikonin in ESCC carcinoma cells, the viability of cells treated with shikonin was measured using MTT assay. As presented in Fig. 1A, B, shikonin and paclitaxel inhibited KYSE270 and KYSE150 cell proliferation at $48 \mathrm{~h}$ following treatment. Although treatments with the single drugs modestly inhibited cell growth, increasing doses of either shikonin (Fig. 1A) or paclitaxel (Fig. 1B) alone did not significantly inhibit KYSE270 and KYSE150 cell growth. The effects of shikonin and paclitaxel co-treatment on KYSE270 and KYSE150 cell proliferation were assessed using various concentrations of paclitaxel, and a low concentration of shikonin at $1 \mu \mathrm{M}$. Significant growth inhibition was observed with 100 or $200 \mu \mathrm{M}$ paclitaxel and $1 \mu \mathrm{M}$ shikonin when compared with cells treated with 100 or $200 \mu \mathrm{M}$ paclitaxel alone (Fig. 1B; $\mathrm{P}<0.001$ ).

Analysis of the effects of shikonin and paclitaxel alone or in combination on KYSE270 and KYSE150 cell proliferation over time were also assessed (Fig. 1C-D). Between 24 and 96 h, cell survival rates were significantly reduced in the combined treatment group (100 $\mathrm{nM}$ paclitaxel and $1 \mu \mathrm{M}$ shikonin) when compared with cell survival in all single agent treatment groups $(\mathrm{P}<0.001)$. These data indicate that shikonin increases paclitaxel-induced cell inhibitory effects in ESCC cells.
Shikonin facilitates mitotic arrest induction caused by paclitaxel. Paclitaxel exerts its cytotoxic effect by interacting with $\beta$-tubulin, which stabilizes the structure of microtubules and prevents the depolymerization of microtubules. This microtubule stabilization leads to cell cycle arrest in the G2/M phase and eventually causes cell death by apoptosis (21). Therefore, the present study determined whether shikonin affects mitotic arrest caused by paclitaxel through flow cytometric analysis. As presented in Fig. 2A-E, following $24 \mathrm{~h}$ of treatment, neither shikonin $(1 \mu \mathrm{M})$ or paclitaxel $(100 \mathrm{nM})$ alone significantly altered the cell cycle compared with the control group. However, compared with treatment with paclitaxel alone, the combined treatment with shikonin and paclitaxel significantly enhanced the cell percentage at the G2/M stage. In addition, to assess the mitotic status of the ESCC cells, the mitotic protein cyclin B was detected. A significant increased protein level of cyclin B was detected following $24 \mathrm{~h}$ of shikonin and paclitaxel combined treatment $(\mathrm{P}<0.05)$, indicating that combined treatment induced mitotic arrest (Fig. 2F).

Shikonin enhances the ability of paclitaxel to induce apoptosis of KYSE270 cells. The present study further assessed the level of cell apoptosis following treatment with either single or combined agents using two methods. First, the percentage of sub-G0 cells was calculated following $72 \mathrm{~h}$ of exposure to the mock treatment, shikonin, paclitaxel, or a combination of shikonin and paclitaxel. As presented in 

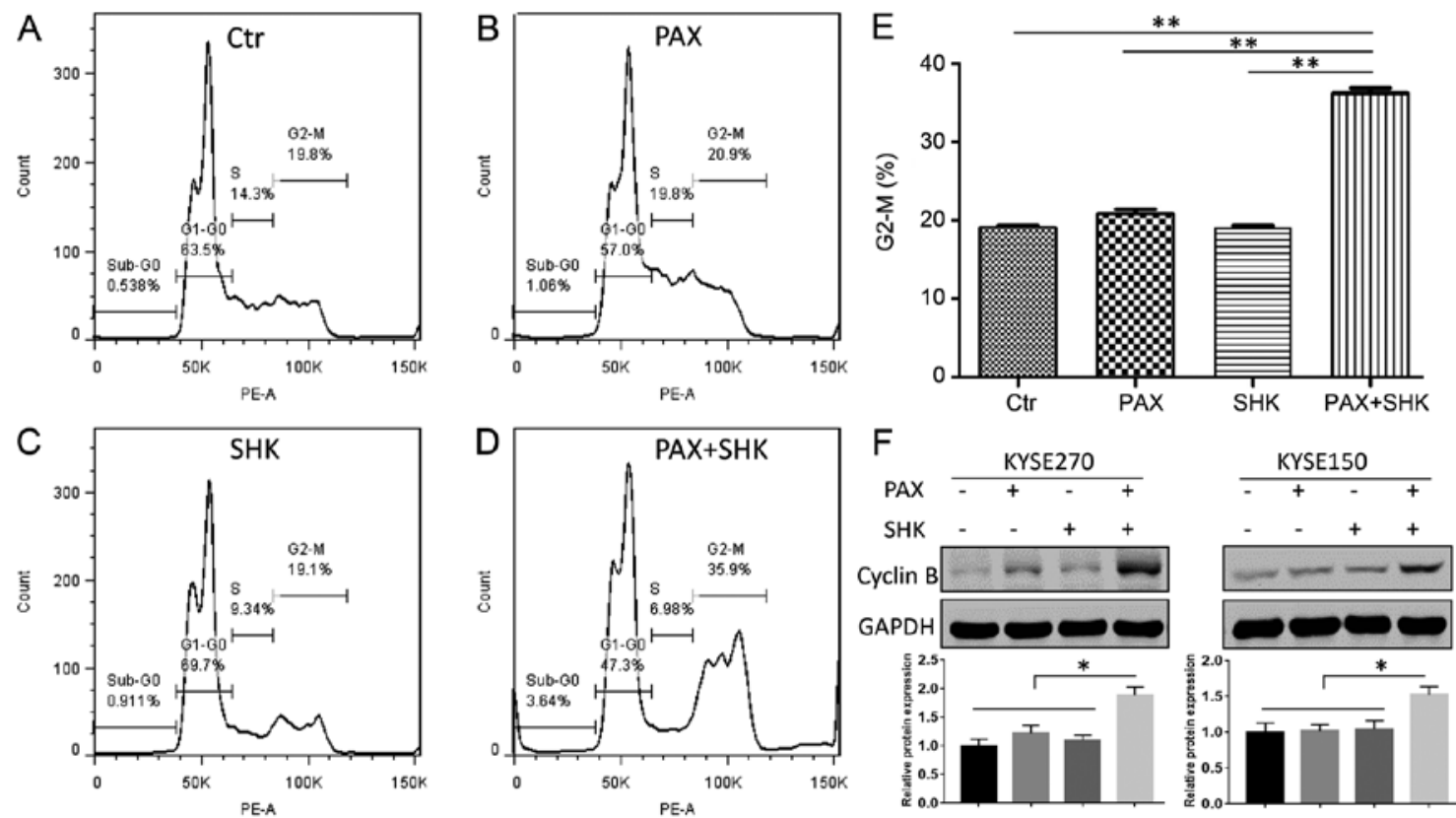

Figure 2. Effects of shikonin and paclitaxel on cell cycle phase distribution. KYSE270 and KYSE150 cells were exposed to $100 \mathrm{nM}$ paclitaxel for $24 \mathrm{~h}$ in either the presence or absence of shikonin $(1 \mu \mathrm{M})$. Cell cycle phase distribution was determined by flow cytometry analysis of (A) control cells, (B) paclitaxel-treated cells, (C) shikonin-treated cells and (D) cells treated with paclitaxel and shikonin. (E) The mitotic cell percentage was calculated based on the percentage of G2-M phase cells in cell cycle analysis. (F) Top panel, the cellular protein expression of cyclin B was analyzed by western blotting. Bottom panel, the blots were quantitated desitometrically and for quantitation of cyclikn B level, data had been normalized by the level of GAPDH. Data were analyzed by one-way ANOVA with Dunnett's multiple comparisons test, and presented as the mean \pm standard error or the mean from three independent experiments. "P<0.05 and ${ }^{* *} \mathrm{P}<0.001$. Ctr, control; PAX, paclitaxel; SHK, shikonin.
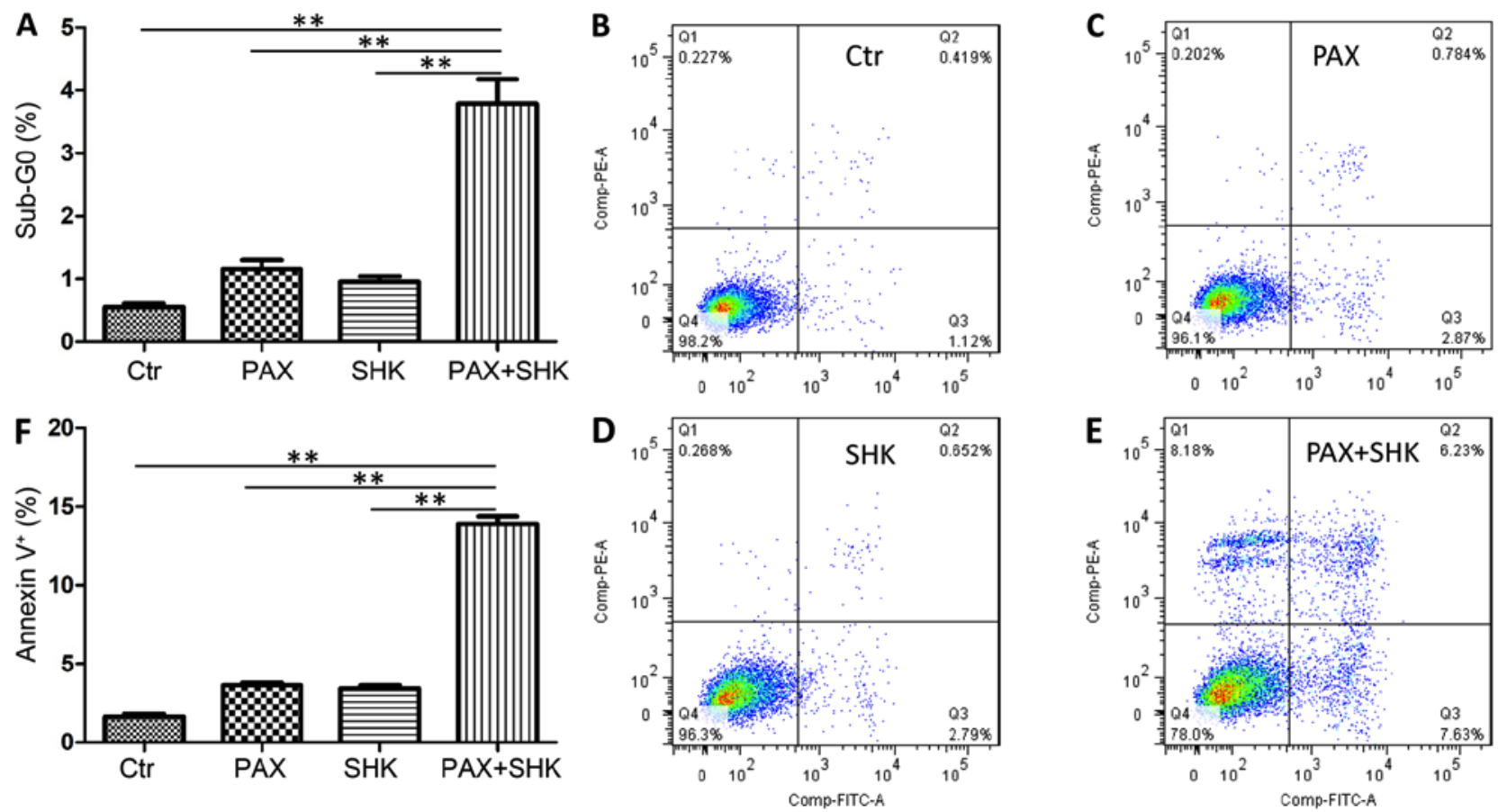

Figure 3. Effects of shikonin on paclitaxel-induced apoptosis. KYSE270 cells were exposed to paclitaxel (100 nM) for $24 \mathrm{~h}$ in either the presence or absence of shikonin $(1 \mu \mathrm{M})$. (A) The percentage of Sub-G0 cells was calculated based on cell cycle data. Flow cytometric analysis of Annexin-V and propidium iodide-stained cells following treatment with (B) DMSO, (C) paclitaxel, (D) shikonin, and (E) paclitaxel and shikonin. (F) The percentage of apoptotic cells in each treatment group. Data were analyzed by one-way ANOVA with Dunnett's multiple comparisons test, and presented as the mean \pm standard error or the mean from three independent experiments. ${ }^{* *} \mathrm{P}<0.001$. Ctr, control; PAX, paclitaxel; SHK, shikonin.

Fig. 3A, the combined treatment significantly increased the cell apoptosis level in KYSE270 cells when compared with both shikonin or paclitaxel-treatment alone $(\mathrm{P}<0.001)$. The present study subsequently detected cell apoptosis through Annexin-V and PI staining. Consistent with the sub-G0 analysis, the shikonin and paclitaxel combined treatment 

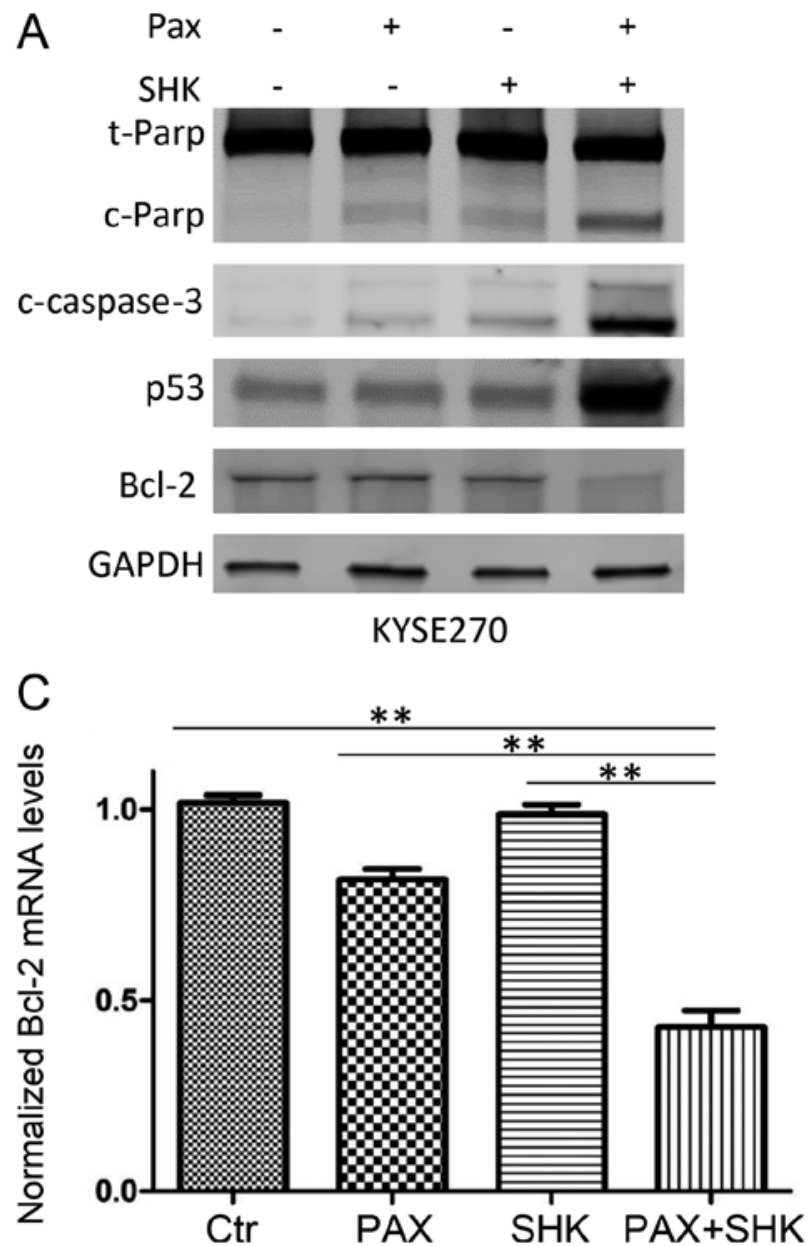
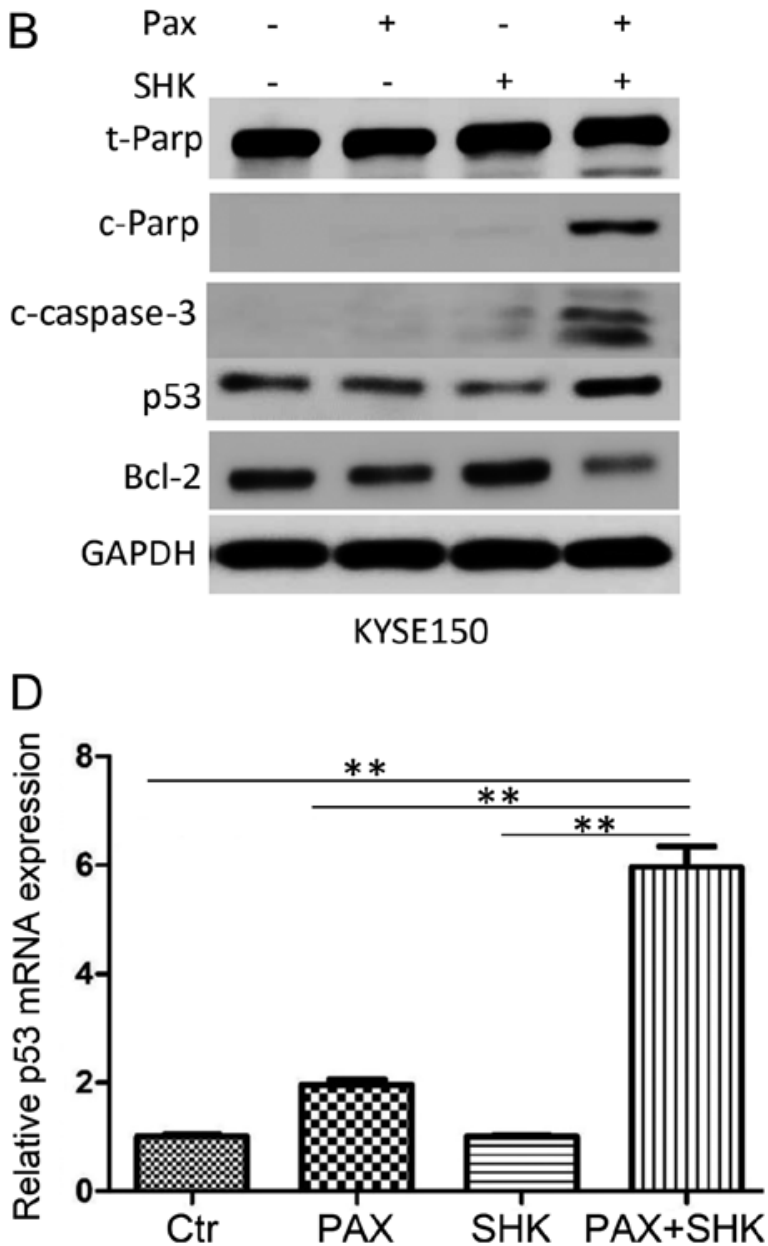

Figure 4. Effects of shikonin and paclitaxel on the expression of apoptosis-associated proteins in ESCC cells. Cells were exposed to $100 \mathrm{nM}$ paclitaxel for $24 \mathrm{~h}$ in either the presence or absence of $1 \mu \mathrm{M}$ shikonin. (A and B) Cellular proteins were analyzed by western blotting. Combined treatment with shikonin and paclitaxel markedly enhanced paclitaxel-induced increased levels of cleaved PARP and cleaved caspase 3, and p53 expression, while it suppressed Bcl-2 expression. Effects of shikonin and pactlitaxel on (C) Bcl-2 and (D) p53 mRNA were analyzed by reverse transcription-quantitative polymerase chain reaction. Significantly altered levels were observed in the combined treatment group compared with all other groups. Data were analyzed by one-way ANOVA with Dunnett's multiple comparisons test, and presented as the mean \pm standard error or the mean from three independent experiments. ${ }^{* *} \mathrm{P}<0.001$. Ctr, control; PAX, paclitaxel; SHK, shikonin; PARP, poly (ADP-ribose) polymerase; c, cleaved; t, total.

significantly increased the percentage of apoptotic cells compared with both shikonin or paclitaxel-treatment alone $(\mathrm{P}<0.001$, Fig. 3B-F $)$.

Shikonin activates caspase-3, increases p53 expression and suppresses Bcl-2 expression. Caspases, particularly caspase-3, are well-established markers of cell apoptosis (22). To further confirm cell apoptosis was induced by various treatments, western blotting was performed to detect cleaved caspase-3 and cleaved PARP. As presented in Fig. 4A and B, while single treatment with paclitaxel only led to low cleaved caspase- 3 and cleaved PARP expression, combined shikonin and paclitaxel treatment markedly enhanced expression levels of cleaved caspase-3 and cleaved PARP.

To further evaluate the mechanism of shikonin promotion of paclitaxel-induced apoptosis in ESCC cells, the present study examined the changes in apoptosis-associated molecules, including p53 and Bcl-2, at the mRNA and protein levels following the indicated drug treatments. The results demonstrated that compared with either shikonin or paclitaxel single treatment, combined treatment significantly reduced
Bcl-2 expression and significantly increased p53 expression $(\mathrm{P}<0.001$, Fig. 4A-D).

\section{Discussion}

The results of the present study demonstrated that shikonin plus paclitaxel combination therapy enhanced the treatment efficacy in ESCC cells. The addition of shikonin to paclitaxel therapy enhanced paclitaxel drug cytotoxicity, which was demonstrated by suppressed cell proliferation. In addition, combined treatment increased cell mitotic arrest, and altered the expression of p53 and Bcl-2, which further affected cell apoptosis.

Paclitaxel is one of the most active agents currently used for the clinical treatment of breast, ovarian, lung, bladder, and head and neck cancer, and has been used for advanced ESCC (23). However, the resistance of cancer cells to paclitaxel is an important issue and can lead to subsequent recurrence and metastasis of malignant tumors $(24,25)$. The synergy of chemotherapy drugs and shikonin can significantly enhance the sensitivity of tumor cells (26). Previous 
studies have demonstrated that the combination of Shikonin and docetaxel or taxotere can enhance drug cytotoxicity in human breast cancer and ovarian carcinoma $(27,28)$. Consistently, the results of the present study indicated that the combination of shikonin and paclitaxel significantly improved effects on ESCC cells. In the present study, MTT results demonstrated that the proliferation inhibition of KYSE270 and KYSE150 cells following treatment with shikonin and paclitaxel was significantly improved compared with either drug alone. Sub-G1 and Annexin-V staining assays demonstrated that paclitaxel could induce apoptosis of KYSE270 and KYSE150 cells; however, the apoptosis rate increased significantly when paclitaxel and shikonin were used in combination. These results were similar to those presented by Yang et al (29) regarding human osteosarcoma. The current study demonstrates that paclitaxel combined with shikonin can dramatically inhibit ESCC cell proliferation and induce cell apoptosis, which supports the potential use of shikonin in ESCC chemotherapy.

The Bcl-2 family consists of more than six anti-apoptotic genes, including $\mathrm{Bcl}-2$, and numerous pro-apoptotic members that regulate cell death and survival $(30,31)$. The overexpression of $\mathrm{Bcl}-2$ protects cancer cells from apoptosis induced by a variety of anticancer agents (32-35). Other studies have also reported that inhibiting or reducing Bcl-2 expression can sensitize cancer cells to chemotherapeutic agents. For example, Bcl-2 inhibitors sensitize leukemia cells to vesicular stomatitis virus oncolysis (36). p53 is a nuclear protein that acts as a tumor suppressor (37). It has been implicated in multiple cellular processes, including inhibition of proliferation (38), mediation of cell cycle arrest (39) and apoptosis (40). Previous studies have demonstrated that p53 can suppress Bcl-2 gene expression transcriptionally by directly binding to the $\mathrm{Bcl}-2$ promoter $(41,42)$. In the present study, while the treatment of shikonin or paclitaxel had little influence on p53 and Bcl-2 expression levels, the combined treatment significantly increased p53 expression, and downregulated $\mathrm{Bcl}-2$ expression, which revealed the role of p53 and Bcl-2 in the mediation of shikonin and paclitaxel-induced apoptosis.

In conclusion, the current study demonstrated that a combination of shikonin and paclitaxel significantly improved cancer cell growth inhibition, altered the expression of apoptosis-associated molecules and enhanced cell apoptosis. The present study indicates that this combination therapy may be investigated as a potential treatment strategy for patients with ESCC. In the future, it may be beneficial to establish primary esophageal cancer cells from patient surgical tissue and perform functional assays on patient derived cells. A patient derived tumor xenograft experiment may also be performed to examine the efficacy and toxicity of combined shikonin and paclitaxel. Finally, future studies should investigate the effect of shikonin on radiation sensitivity and chemo-radiosensitivity.

\section{Acknowledgements}

The authors would like to thank Dr Hui Zhang (Sun Yat-Sen University Cancer Center, Guangzhou, China) for providing the KYSE270 and KYSE150 cell lines used in the present study.

\section{Funding}

No funding was received.

\section{Availability of data and materials}

The datasets used and/or analyzed during the present study are available from the corresponding author on reasonable request.

\section{Authors' contributions}

JL, WD and XH designed the research. WD, XH, ZY, YW and $\mathrm{XZ}$ performed the experiments. JL wrote the manuscript. All authors read and approved the final manuscript.

\section{Ethics approval and consent to participate}

Not applicable.

\section{Patient consent for publication}

Not applicable.

\section{Competing interests}

The authors declare that they have no competing interests.

\section{References}

1. Siegel RL, Miller KD and Jemal A: Cancer statistics, 2019. CA Cancer J Clin 69: 7-34, 2019.

2. Zhang Y: Epidemiology of esophageal cancer. World J Gastroenterol 19: 5598-5606, 2013.

3. Chen W, Zheng R, Baade PD, Zhang S, Zeng H, Bray F, Jemal A, Yu XQ and He J: Cancer statistics in China, 2015. CA Cancer J Clin 66: 115-132, 2016.

4. Law S and Wong J: Changing disease burden and management issues for esophageal cancer in the Asia-Pacific region. J Gastroenterol Hepatol 17: 374-381, 2002.

5. Holmes RS and Vaughan TL: Epidemiology and pathogenesis of esophageal cancer. Semin Radiat Oncol 17: 2-9, 2007.

6. Stanton RA, Gernert KM, Nettles JH and Aneja R: Drugs that target dynamic microtubules: A new molecular perspective. Med Res Rev 31: 443-481, 2011.

7. Yvon AM, Wadsworth P and Jordan MA: Taxol suppresses dynamics of individual microtubules in living human tumor cells. Mol Biol Cell 10: 947-959, 1999.

8. Blagosklonny MV: Prolonged mitosis versus tetraploid checkpoint: How p53 measures the duration of mitosis. Cell Cycle 5: 971-975, 2006.

9. Dumontet $\mathrm{C}$ and Jordan MA: Microtubule-binding agents: A dynamic field of cancer therapeutics. Nat Rev Drug Discov 9: 790-803, 2010.

10. Ikui AE, Yang CP, Matsumoto T and Horwitz SB: Low concentrations of taxol cause mitotic delay followed by premature dissociation of p55CDC from Mad2 and BubR1 and abrogation of the spindle checkpoint, leading to aneuploidy. Cell Cycle 4: 1385-1388, 2005.

11. Orr GA, Verdier-Pinard P, McDaid $\mathrm{H}$ and Horwitz SB: Mechanisms of Taxol resistance related to microtubules. Oncogene 22: 7280-7295, 2003.

12. Hou Y, Guo T, Wu C, He X and Zhao M: Effect of shikonin on human breast cancer cells proliferation and apoptosis in vitro. Yakugaku Zasshi 126: 1383-1386, 2006.

13. Han W, Li L, Qiu S, Lu Q, Pan Q, Gu Y, Luo J and Hu X: Shikonin circumvents cancer drug resistance by induction of a necroptotic death. Mol Cancer Ther 6: 1641-1649, 2007.

14. Yang H, Zhou P, Huang H, Chen D, Ma N, Cui QC, Shen S, Dong W, Zhang X, Lian W, et al: Shikonin exerts antitumor activity via proteasome inhibition and cell death induction in vitro and in vivo. Int J Cancer 124: 2450-2459, 2009. 
15. Chang IC, Huang YJ, Chiang TI, Yeh CW and Hsu LS: Shikonin induces apoptosis through reactive oxygen species/extracellular signal-regulated kinase pathway in osteosarcoma cells. Biol Pharm Bull 33: 816-824, 2010.

16. Long S, GuangZhi Y, BaoJie G, Wei X, YanYong H, YingLi W, Yang $\mathrm{Z}$ and LiHua L: Shikonin derivatives protect immune organs from damage and promote immune responses in vivo in tumour-bearing mice. Phytother Res 26: 26-33, 2012.

17. Zhao Q, Assimopoulou AN, Klauck SM, Damianakos H, Chinou I, Kretschmer N, Rios JL, Papageorgiou VP, Bauer R and Efferth T: Inhibition of c-MYC with involvement of ERK/JNK/MAPK and AKT pathways as a novel mechanism for shikonin and its derivatives in killing leukemia cells. Oncotarget 6: 38934-38951, 2015

18. Liu X and Sun G: Shikonin enhances Adriamycin antitumor effects by inhibiting efflux pumps in A549 cells. Oncol Lett 14: 4270-4276, 2017.

19. Song J, Zhao Z, Fan X, Chen M, Cheng X, Zhang D, Wu F, Ying $X$ and Ji J: Shikonin potentiates the effect of arsenic trioxide against human hepatocellular carcinoma in vitro and in vivo. Oncotarget 7: 70504-70515, 2016.

20. Livak KJ and Schmittgen TD: Analysis of relative gene expression data using real-time quantitative PCR and the 2(-Delta Delta C(T)) method. Methods 25: 402-408, 2001

21. Mukhtar E, Adhami VM and Mukhtar H: Targeting microtubules by natural agents for cancer therapy. Mol Cancer Ther 13 275-284, 2014

22. Elmore S: Apoptosis: A review of programmed cell death. Toxicol Pathol 35: 495-516, 2007.

23. Henley D, Isbill M, Fernando R, Foster JS and Wimalasena J: Paclitaxel induced apoptosis in breast cancer cells requires cell cycle transit but not Cdc2 activity. Cancer Chemother Pharmacol 59: 235-249, 2007.

24. Januchowski R, Zawierucha P, Andrzejewska M, Ruciński M and Zabel M: Microarray-based detection and expression analysis of $\mathrm{ABC}$ and SLC transporters in drug-resistant ovarian cancer cell lines. Biomed Pharmacother 67: 240-245, 2013.

25. Sun QL, Sha HF, Yang XH, Bao GL, Lu J and Xie YY: Comparative proteomic analysis of paclitaxel sensitive A549 lung adenocarcinoma cell line and its resistant counterpart A549-Taxol. J Cancer Res Clin Oncol 137: 521-532, 2011.

26. Zhao Q, Kretschmer N, Bauer R and Efferth T: Shikonin and its derivatives inhibit the epidermal growth factor receptor signaling and synergistically kill glioblastoma cells in combination with erlotinib. Int J Cancer 137: 1446-1456, 2015.

27. Li W, Liu J, Jackson K, Shi R and Zhao Y: Sensitizing the therapeutic efficacy of taxol with shikonin in human breast cancer cells. PLoS One 9: e94079, 2014.

28. Wang Z, Yin J, Li M, Shen J, Xiao Z, Zhao Y, Huang C, Zhang H, Zhang Z, Cho $\mathrm{CH}$ and Wu X: Combination of shikonin with paclitaxel overcomes multidrug resistance in human ovarian carcinoma cells in a P-gp-independent manner through enhanced ROS generation. Chin Med 14: 7, 2019.

29. Yang Q, Li S, Fu Z, Lin B, Zhou Z, Wang Z, Hua Y and Cai Z: Shikonin promotes adriamycininduced apoptosis by upregulating caspase-3 and caspase- 8 in osteosarcoma. Mol Med Rep 16: 1347-1352, 2017.
30. Danial NN and Korsmeyer SJ: Cell death: Critical control points. Cell 116: 205-219, 2004.

31. Aravind L, Dixit VM and Koonin EV: Apoptotic molecular machinery: Vastly increased complexity in vertebrates revealed by genome comparisons. Science 291: 1279-1284, 2001.

32. Ogura T, Tanaka Y, Tamaki H and Harada M: Docetaxel induces $\mathrm{Bcl}-2$ - and pro-apoptotic caspase-independent death of human prostate cancer DU145 cells. Int J Oncol 48: 2330-2338, 2016.

33. Rong YP, Barr P, Yee VC and Distelhorst CW: Targeting Bcl-2 based on the interaction of its $\mathrm{BH} 4$ domain with the inositol 1,4,5-trisphosphate receptor. Biochim Biophys Acta 1793: 971-978, 2009.

34. Srivastava RK, Sasaki CY, Hardwick JM and Longo DL: Bcl-2-mediated drug resistance: Inhibition of apoptosis by blocking nuclear factor of activated $\mathrm{T}$ lymphocytes (NFAT)-induced Fas ligand transcription. J Exp Med 190: 253-265, 1999.

35. Yang T, Xu F, Sheng Y, Zhang W and Chen Y: A targeted proteomics approach to the quantitative analysis of ERK/Bcl-2-mediated anti-apoptosis and multi-drug resistance in breast cancer. Anal Bioanal Chem 408: 7491-7503, 2016.

36. Samuel S, Beljanski V, Van Grevenynghe J, Richards S, Ben Yebdri F, He Z, Nichols C, Belgnaoui SM, Steel C, Goulet ML, et al: BCL-2 inhibitors sensitize therapy-resistant chronic lymphocytic leukemia cells to VSV oncolysis. Mol Ther 21: 1413-1423, 2013.

37. Smith ND, Rubenstein JN, Eggener SE and Kozlowski JM: The p53 tumor suppressor gene and nuclear protein: Basic science review and relevance in the management of bladder cancer J Urol 169: 1219-1228, 2003.

38. Xiong Y, Hannon GJ, Zhang H, Casso D, Kobayashi R and Beach D: p21 is a universal inhibitor of cyclin kinases. Nature 366: 701-704, 1993.

39. Yew PR and Berk AJ: Inhibition of p53 transactivation required for transformation by adenovirus early $1 \mathrm{~B}$ protein. Nature 357 : 82-85, 1992.

40. Yonish-Rouach E, Deguin V, Zaitchouk T, Breugnot C, Mishal Z, Jenkins JR and May E: Transcriptional activation plays a role in the induction of apoptosis by transiently transfected wild-type p53. Oncogene 11: 2197-2205, 1995.

41. Wu Y, Mehew JW, Heckman CA, Arcinas M and Boxer LM: Negative regulation of bcl-2 expression by $\mathrm{p} 53$ in hematopoietic cells. Oncogene 20: 240-251, 2001

42. May P and May E: Twenty years of p53 research: structural and functional aspects of the p53 protein. Oncogene 18: 7621-7636, 1999.

This work is licensed under a Creative Commons Attribution-NonCommercial-NoDerivatives 4.0 International (CC BY-NC-ND 4.0) License. 